\begin{tabular}{|c|c|}
\hline 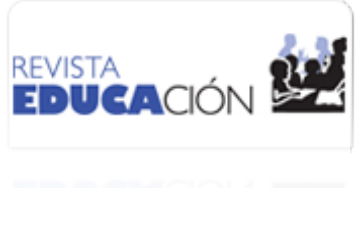 & $\begin{array}{l}\text { Revista Educación } \\
\text { ISSN: 0379-7082 } \\
\text { ISSN: } 2215-2644 \\
\text { revedu@gmail.com } \\
\text { Universidad de Costa Rica } \\
\text { Costa Rica }\end{array}$ \\
\hline
\end{tabular}

\title{
¿Cómo se conceptualizan las ayudantías? La voz de las personas tutoras universitarias
}

\author{
Cabrera-Murcia, E. Piedad \\ ¿Cómo se conceptualizan las ayudantías? La voz de las personas tutoras universitarias \\ Revista Educación, vol. 44, núm. 1, 2020 \\ Universidad de Costa Rica, Costa Rica \\ Disponible en: http://www.redalyc.org/articulo.oa?id=44060092015 \\ DOI: https://doi.org/10.15517/revedu.v44i1.33357
}

Esta obra está bajo una Licencia Creative Commons Atribución-NoComercial-SinDerivar 3.0 Internacional. 


\title{
¿Cómo se conceptualizan las ayudantías? La voz de las personas tutoras universitarias
}

How is Peer Tutoring Conceptualized? The Voice of University Tutors

E. Piedad Cabrera-Murcia

Universidad Alberto Hurtado, Chile

pcabrerm@uahurtado.cl

(iD http://orcid.org/0000-0003-1122-5232
DOI: https://doi.org/10.15517/revedu.v44i1.33357

Redalyc: http://www.redalyc.org/articulo.oa?id=44060092015

Recepción: 07 Febrero 2019

Aprobación: 04 Octubre 2019

\begin{abstract}
Resumen:
El presente estudio aborda la comprensión del significado de las ayudantías desde la perspectiva de las personas ayudantes de las carreras de Psicología e Ingeniería Comercial de la Universidad Alberto Hurtado en Chile. Se utilizó una metodología cualitativa enmarcada en la grounded theory. Participó una muestra intencionada de 12 estudiantes pertenecientes a ambas carreras, quienes llevaban por lo menos un año en el rol de ayudantes de pregrado, con evaluaciones positivas desde la mirada del estudiantado tutorado. Se recogieron los datos a partir de entrevistas semiestructuradas, desarrollando codificación abierta y axial buscando patrones de relación que permitiesen construir el significado que le otorgan a este espacio los propios ayudantes. Los resultados evidencian una forma particular de concebir el espacio de ayudantía, las condiciones consideradas como necesarias para que se produzca el aprendizaje y las estrategias usadas en su desarrollo. El análisis relacional destaca la ayudantía como un espacio de aprendizaje recíproco, el cual se ve influido por factores que facilitan o inhiben la posibilidad de aprender de forma colectiva, así como estrategias de acción que potencian esta forma de aprender. Para concluir, se releva las ayudantías como un método de aprendizaje y enseñanza, que favorece el aprender y enseñar a aprender.
\end{abstract}

Palabras ClaVE: Aprendizaje, Tutoría entre pares, Enseñanza, Universidad.

\section{Abstract:}

This study addresses how peer tutoring is understood from the perspective of tutors for the Psychology and Commercial Engineering programs at the Alberto Hurtado University in Chile. A qualitative grounded theory methodology was used with an intended sample of 12 students from both majors who had been undergraduate tutors for at least one year and had received positive reviews by their peers. Data was gathered based on semi-structured interviews, development of open and axial coding and seeking relationship patters to better understand the meaning of peer tutoring and the necessary conditions for learning and strategies used to develop this process. A relational analysis highlights tutoring as an opportunity for reciprocal learning influenced by factors that facilitate or inhibit the possibility of collective learning, as well as action strategies to foster this type of learning. To conclude, peer tutoring is considered to be another method of learning that favors teaching and teaches learning. Keys Words: Learning, Peer Tutoring, Teaching, University. Fecha de recibido: 07-02-2019 Fecha de aceptado: 04/10/2019 Agradecimientos A las personas ayudantes que participaron de esta investigación, así como a las carreras de Psicología e Ingeniería Comercial de esta casa de estudios. Introducción

KEYWORDS: Learning, Peer Tutoring, Teaching, University.

\section{INTRODUCCIÓN}

Actualmente, la pregunta sobre cómo aprende el estudiantado universitario es objeto de debate, análisis y de propuestas de acción a implementar, direccionadas desde estamentos gubernamentales o universitarios, o bien desde el profesorado interesado en mejorar sus prácticas pedagógicas. Dicha pregunta, remite a pensar sobre cómo se enseña, sobre las formas o métodos que brinden oportunidades de aprendizaje enriquecedoras para la diversidad de estudiantes que actualmente ingresan a la educación superior (Gallardo y Reyes, 2010).

Para la Organización para la Cooperación y el Desarrollo Económico (OCDE, 2009) las universidades en Chile deberían caminar hacia el uso de métodos los cuales favorezcan el tránsito entre formas de enseñar centradas en la transmisión, por otras focalizadas en el aprendizaje activo del estudiantado, que le aseguren un 
aprendizaje significativo aplicable en su futuro campo laboral. Lo anterior ha involucrado mayoritariamente al profesorado y al alumnado al interior de las aulas universitarias, dejando de lado otros espacios en los cuales se producen interacciones que, potencialmente pueden producir aprendizajes significativos.

Las ayudantías, se constituyen en uno de estos espacios, en los cuales cada estudiante de un semestre superior, que demuestran experticia y motivación en un área disciplinar, puedan enseñar a sus compañeras y compañeros de semestres inferiores. Se estima que, al haber cursado previamente la actividad curricular en la que apoyará el aprendizaje de sus pares, las personas ayudantes podrían reconocer qué conocimientos, habilidades y formas de razonar son útiles en pro de alcanzar los propósitos de aprendizaje.

Asimismo, se espera que el grupo de estudiantes aprenda sobre el campo disciplinar en cuestión y que reflexionen sobre cómo se aprende, gracias a las formas de aproximarse al aprendizaje, que brindan ayudantes al enseñar. Para estos últimos, este espacio les ofrece confirmaciones, reestructuraciones o complementos en su propio aprendizaje, que les lleva a la reflexión y mejora continua. Por tanto, la interacción que se produce entre ayudante y estudiante, puede considerarse un método de enseñanza, y además de aprendizaje, pues este se produciría de forma bidireccional (Biggs, 2005).

Si se reconocen las ayudantías como un espacio que favorece la construcción de aprendizajes, cabe preguntarse ¿cuál es el valor agregado que se produce al aprender entre pares? ¿Cómo se benefician las personas ayudantes? ¿Cómo se benefician quienes reciben las tutorías? ¿De qué manera las estrategias usadas por cada ayudante impactan los procesos de aprender y enseñar a aprender? ¿Cómo puede usarse esta información en el planteamiento de un programa de formación dirigido a ayudantes?

En el contexto de este estudio, así como en las universidades que hacen uso de la figura del ayudante, estas preguntas cobran sentido, pues su rol es el de asistir al profesorado en la ejecución de actividades de laboratorio, reforzamiento o ejercitación de contenidos con el fin de acompañar el aprendizaje del estudiantado (Cabrera, 2017a; Universidad Católica del Maule, 2007; Universidad de Santiago de Chile [USACH], 2009; Universidad Diego Portales [UDP], 2011; Universidad del Desarrollo [UDD], 2014).

Está claro que aprender va más allá del diseño de clases magistrales, de la toma de apuntes o incluso de preparaciones para aprobar pruebas o exámenes (Doyle, 2008). Si esto es así, es evidente que al igual que en el aula, las ayudantías deben ser pensadas -diseñadas- como nichos de aprendizaje en que, las personas ayudantes y el estudiantado, movilizan sus recursos cognitivos en beneficio de la creación de zonas colectivas de desarrollo próximo (Engeström, 2001).

Las ayudantías, por tanto, son espacios en los cuales ayudantes y sus pares reconstruyen sus aprendizajes, y aprenden profunda y no superficialmente. Estas zonas colectivas, de las que habla Engeström, se construyen si se usan estrategias en las cuales se ponen en juego, formas de colaboración, de comprensión, de construcción o complementariedad de rutas de razonamiento. Esta forma de conceptualizar las ayudantías, los convierte en un nicho que potencia el aprendizaje del estudiantado tutorado, de ayudantes, así como un espacio en el cual se hace uso de ciertas estrategias que favorecen el aprendizaje recíproco.

La necesidad de contar a nivel universitario con métodos que aseguren un aprendizaje profundo en el estudiantado, y considerando la ayudantía como aquel que puede favorecer esta forma de aprender, este estudio pretende describir, desde la percepción del propio ayudante, el impacto que estas tienen en los procesos de aprender y enseñar a aprender, tanto en las personas ayudantes como en el estudiantado.

Para ello, esta investigación se centra en evidenciar cómo se concibe esta forma de aprender desde la voz de los propios ayudantes, identificando aquellas condiciones y estrategias que a su juicio son beneficiosas para sus pares y para sí mismos, en su proceso de aprendizaje. Teóricamente el estudio es relevante pues permite abrir un espacio de conocimiento que no ha sido estudiado, o bien visible, en relación con las ayudantías como un método de aprendizaje y de enseñanza que apoya el aprendizaje del estudiantado tutorado, así como de ayudantes. 


\section{ANTECEDENTES CONTEXTUALES Y REFERENTES CONCEPTUALES}

En Chile el estudio de la figura de las personas ayudantes en la universidad, aún no cuenta con un cuerpo de conocimiento sólido y extendido, pese a que, su rol es clave en el acompañamiento de la trayectoria académica de cada tutorado. Algunas investigaciones, permiten visibilizarlo como un/a estudiante de curso superior que ayuda al estudiantado tutorado a alcanzar aprendizajes en el proceso de enseñar a aprender en el que participan (Cabrera, 2017b; Sánchez, Sanmartín, Mardones y Fauré, 2017).

Resulta paradójico, que existiendo la figura del ayudante en el ámbito de la educación superior se sepa poco -o de manera difusa- sobre su relevancia como método que favorece el aprendizaje del estudiantado en la universidad. Si bien, desde algunas unidades de docencia de universidades en Chile se ha empezado a mirar con detención esta figura, aun se requiere investigación que brinde información en relación con el valor agregado que otorga este actor en la consecución de la trayectoria formativa del estudiantado que ha sido acompañado en algún momento de su proceso de aprendizaje.

Finalmente, uno de los desafíos actuales en las universidades de Chile, está en poder acompañar los procesos formativos de quienes ingresan. En especial, porque los cambios estructurales al sistema, suscitados por ejemplo por la ley de gratuidad, deben venir aparejadas a oportunidades educativas de calidad. Promulgar educación gratuita invita a la universidad a pensar formas de acompañar las trayectorias formativas, de tal manera de acortar las brechas del estudiantado que son primera generación de ingreso a la universidad.

Bajo este escenario, es prioritario entender cómo formas de enseñar a aprender, creadas como apoyo a la gestión del personal docente, pueden ser vistas con un lente más amplio. Un lente que permite visualizarlas como formas que favorecen el aprender de manera conjunta, entre estudiantes ayudantes y tutorados. En las carreras de la Universidad Alberto Hurtado, la figura del ayudante es clave en los procesos formativos del estudiantado y por ello, se torna prioritario profundizar sobre el rol que esta figura desempeña, y los significados que ellos mismos les otorgan a los espacios de ayudantía.

A nivel internacional, estos énfasis han sido objeto de análisis. Países anglosajones han centrado sus esfuerzos en desarrollar acciones de formación e inducción a graduados, que ejercen el rol de ayudantes en cursos de formación de pregrado (Gerton, 2016; Kajfez, Matusovich y Lee, 2016; Melton, 2016). Investigaciones recientes, se han focalizado en ayudantes que son estudiantes de pregrado, actores que han emergido en aquellas universidades de tamaño medio con pocos programas de postgrado (Filz y Gurung, 2013).

Aun cuando se perfilan diferencias en términos de formación y funciones entre el ayudante graduado vs. el ayudante de pregrado, el estudiantado que asiste a ayudantías con este último grupo reporta avances en la construcción de sus aprendizajes y, además, las personas ayudantes reportan logros para sí mismos (Gordon, Henry y Michaux, 2013; Hogan, Norcross, Cannon y Karpiak, 2007; Philipp, Tretter y Rich, 2016a; Sari, Çeliköz y Ünal, 2017).

En diferentes estudios las ayudantías son percibidas, desde el estudiantado, como fuente de evaluación continua que permite la construcción de sus productos, y mejora de habilidades (Alrajhi y Aldhafri; 2015; Malm, Bryngfors y Mörner, 2015). En otros, se explicitan incrementos en el rendimiento académico medido en notas, así como la identidad del estudiantado con la disciplina enseñada (Lindsay, Carlsen, Boaz, y Marshall, 2017; Philipp, Tretter y Rich, 2016b; Sari et al, 2017).

Otros estudios, relevan características distintivas al querer definir a una persona ayudante accesible y como un apoyo al aprendizaje. El entusiasmo que muestra sobre la materia y la enseñanza (Alrajhi y Aldhafri, 2015), o bien, el ser un comunicador efectivo, flexible y de mente abierta en las ayudantías, establecer buenas relaciones al interior del grupo, con conocimientos tecnológicos, con capacidad para proveer retroalimentación inmediata del trabajo, tanto como tener conocimiento del contenido del curso (Filz y Gurung, 2013). Características relevantes si se piensa en cómo elegir ayudantes o bien, en cómo capacitarlos. 
En esta misma línea, las personas ayudantes reportan haber obtenido aprendizajes profundos al ejecutar ayudantías. Particularmente sobre contenidos y habilidades propias de la disciplina, en formas de enseñar, en aplicar lo aprendido a su propia experiencia como estudiantes, en mayor seguridad, en habilidades para entender la perspectiva de otros, tanto como en habilidades comunicativas (Deshler, 2015; McKeegan, 1998, Odon, Ho y Moore, 2015; Philipp et al., 2016a; Weidert, Wendorf, Gurung y Filz, 2012).

De este conjunto de habilidades, se sustentan las estrategias que las personas ayudantes usan para desarrollar el acompañamiento a sus pares. Enseñar al estudiantado de forma participativa, haciendo uso por ejemplo de preguntas divergentes, comunicar de forma clara las ideas logrando que lo complejo sea más manejable, usar ejemplos como herramienta útil para el aprendizaje de nuevos conceptos, así como el ser paciente y poder establecer un buen clima para el aprendizaje, destacan como aquellas estrategias que ayudantes implementan en beneficio del aprendizaje de sus pares (Filz y Gurung, 2013; Odon et al., 2015; Philipp et al., 2016a).

Las investigaciones señaladas no solo dan cuenta de la necesidad de formar al estudiantado que quieren apoyar el aprendizaje de sus pares, sino que evidencian la emergencia de considerar ciertas condiciones que aseguren el buen funcionamiento de las mismas. Particularmente, dialogar sobre cómo propiciar buenas prácticas, entregar apoyo y acompañamiento en la implementación de estrategias, potenciar en ayudantes conocimientos y habilidades propios de las actividades curriculares, así como modelar un pensamiento reflexivo y crítico sobre su ejercicio de enseñanza (Odon et al., 2015; Philipp et al., 2016a; Sana, Pachai y Kim, 2011).

A la luz de estos antecedentes contextuales y empíricos expuestos anteriormente, resulta evidente ampliar la comprensión que se tiene de la ayudantía como método de enseñanza-aprendizaje. La consideración de los propios involucrados, resulta clave para comprender las significaciones que estos tienen respecto a las ayudantías.

\section{Procedimientos metodológicos}

Ayudantes de las carreras de Ingeniería y de Psicología de la Universidad Alberto Hurtado, en Santiago de Chile, participaron de este estudio. La universidad, de carácter público, acompaña a 5.461 estudiantes en su trayectoria formativa en programas de humanidades, ciencias sociales y educación. Desde las carreras que conforman la Universidad, se promueve el que, el estudiantado de semestres superiores apoye a sus compañeros de semestres previos. Si bien, existe este acompañamiento, se requiere indagar sobre su construcción, y el impacto de este en los procesos formativos. Razón que motivó el estudio, pues era imperativo conocer desde las voces de quienes elaboran la tarea de acompañamiento de la trayectoria formativa, cómo conciben estos espacios de enseñanza-aprendizaje.

Para ello, la presente investigación adoptó una metodología cualitativa de carácter interpretativo (Creswell, 2014; Flick, 2004) orientado a comprender el significado que, desde la perspectiva de las personas entrevistadas, les atribuyen a las ayudantías. Se aplicaron los procedimientos metodológicos de la Grounded Theory de Glaser y Strauss (Strauss y Corbin, 2002), pues estos permiten, a partir de las experiencias subjetivas de cada participante, acceder a las significaciones de las ayudantías como objeto de estudio.

La muestra estuvo compuesta por 12 ayudantes, 7 de la carrera de Ingeniería Comercial y 5 de Psicología. El rango promedio de edades de 20 años. Teniendo en cuenta el objetivo del estudio, se intencionó que estos impartieran ayudantías por lo menos durante 1 año y que hubiesen recibido una evaluación positiva de sus pares, a través del componente ayudantías de la evaluación docente, el cual se aplica al término del semestre.

En cuanto al procedimiento de selección de la muestra, recolección y análisis de los datos, se desarrollaron en forma paralela y no en fases sucesivas (Strauss y Corbin, 2002). Para la muestra, se contactó a un informante clave de la carrera, cuyo criterio era identificar aquellos ayudantes que fuesen evaluados de 
forma positiva por sus pares. Se desarrollaron entrevistas semiestructuradas (Flick, 2004), con la finalidad de comprender su concepción sobre las ayudantías como un espacio de enseñanza-aprendizaje.

A cada participante se le invitó a establecer un diálogo a partir de preguntas generadoras, con la finalidad de profundizar en sus concepciones y sobre los beneficios que trae para la construcción de aprendizajes en sus pares, y para sí mismos. Se plantearon preguntas sensibilizadoras (¿Por qué crees tú, el estudiantado tutorado indicó como aspecto positivo en tus ayudantías que explicabas bien?); así como preguntas teórico-prácticas ¿Qué elementos consideras al momento de diseñar tu ayudantía? (Strauss y Corbin, 2002). Finalmente, todos fueron informados sobre el propósito del estudio, y quienes participaron consignaron su firma a través del consentimiento informado.

El análisis de la información se elaboró siguiendo los procedimientos metodológicos propuestos por la grounded theory. Una codificación abierta permitió ejecutar un análisis descriptivo de la información, y a partir de este, levantando las categorías, que se fueron articulando descriptiva y analíticamente. La Tabla 1 , ejemplifica el proceso de categorización para la categoría de estrategias, particularmente el uso de ejercicios. Como se muestra, algunas preguntas de la entrevista favorecieron la obtención de respuestas asociadas a las estrategias que los ayudantes usan en beneficio de la construcción de aprendizajes. A esta categoría se asoció sus propiedades y dimensiones, las que definen y caracterizan.

TABLA 1

Desarrollo de la categoría de uso de ejercicios

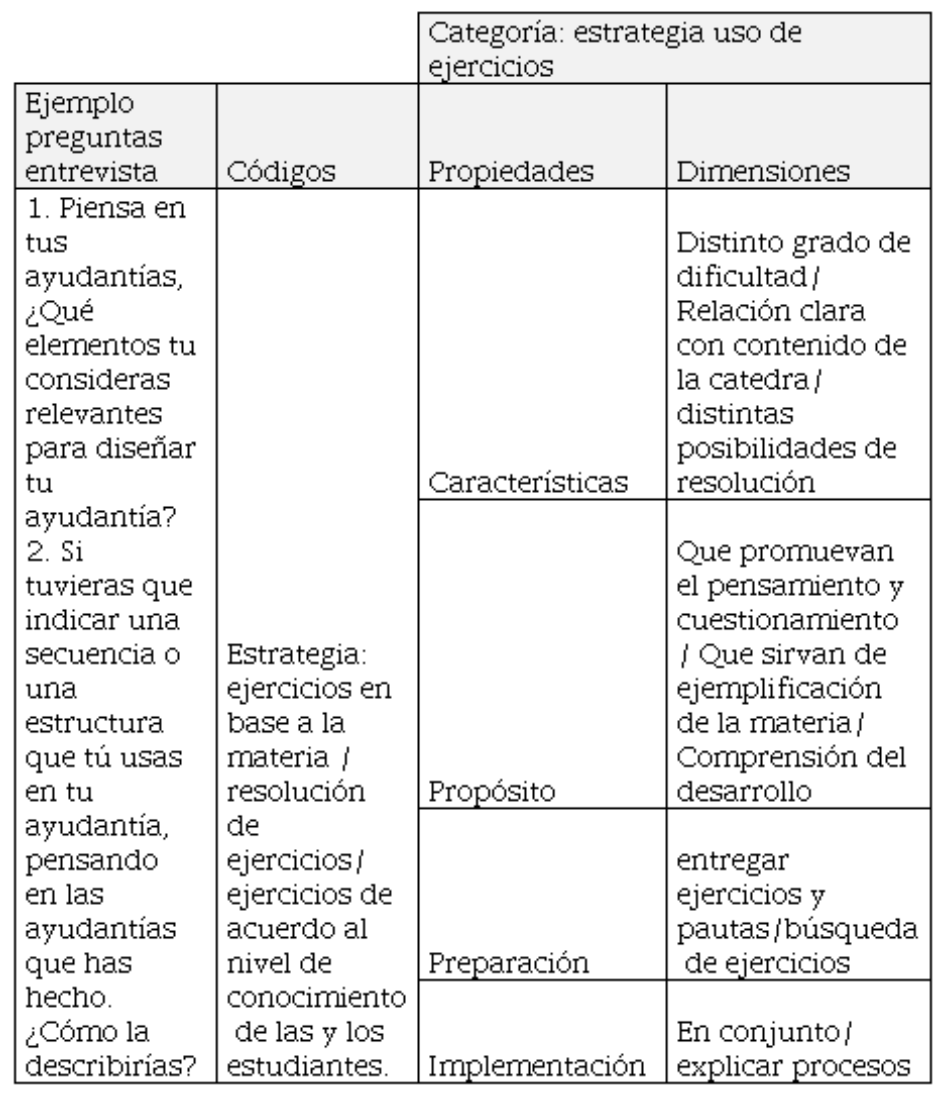

Fuente: Elaboración propia

En este estudio, se recogieron los datos desde las personas ayudantes de dos carreras diferentes, usando así una triangulación de fuentes de tal manera de aumentar la confiabilidad y validez de los resultados. Los datos fueron recogidos por dos entrevistadores distintos, y codificados -de manera independiente-, por dos 
investigadores provenientes de la educación y la psicología, y que compartían en su perfil la formación y experiencia en el área de la psicología educacional.

Se optó también, por la triangulación de investigadores, con la finalidad de disminuir el sesgo en la recopilación como en el análisis de los datos de este estudio (Denzin, 1989). Esto permitió contar con análisis independientes que, posteriormente, fueron comparados obteniendo la conceptualización del espacio de ayudantías desde los propios ayudantes.

\section{Resultados}

Un análisis descriptivo evidencia, a partir de la triangulación de los datos y la codificación de los investigadores, la emergencia de tres categorías orientadoras del discurso de las personas participantes. Estos hallazgos muestran las similitudes encontradas, en el discurso de ayudantes de ambas carreras, en cómo se conceptualiza la ayudantía y las condiciones que la caracterizan, así como algunas distinciones asociadas a la disciplina. Un análisis relacional, presenta a las ayudantías como un espacio de aprendizaje recíproco, fenómeno emergente en este estudio.

\section{Análisis descriptivo}

\section{Concepción sobre el espacio de ayudantia}

La ayudantía, para los participantes de ambas carreras, se conceptualiza inicialmente como un lugar de reflexión que ofrece oportunidades de visibilizar el pensamiento, de planteamiento de preguntas las cuales invitan al estudiantado tutorado a tener un rol activo en su proceso de aprendizaje:

... pienso que la ayudantía como espacio es eso: ayudar a pensar un poquito más... más bien plantear preguntas para movilizar al estudiante a que el busque también (Alan, comunicación personal, 2014)

... que ellos traten de pensar por ellos mismos... no tratar de hacer siempre lo mismo, darles una idea, que ellos aprendan el método correspondiente, la forma que se quiere desarrollar o solucionar los problemas para que ellos puedan después utilizar esas mismas herramientas, pero a partir de su propio pensamiento... (Germán, comunicación personal, 2014)

Una segunda concepción, refiere a considerar la ayudantía como un espacio de construcción de conocimiento y de comprensión de aquello que se quiere enseñar. Aquí se habla de entablar diálogos, de movilizar el pensamiento:

... Yo me hago siempre preguntas y trato de interactuar, cuando hago las ayudantías, siempre hago las preguntas de quien no entiende, qué les tengo que enseñar para que entiendan. (Joaquín, comunicación personal, 2014)

Entonces trato de entablar un dialogo, de alguna manera hablarles, susurrarles al oído y decirles alguna cosa o no sé, decirles: Bueno, ¿Quépiensan de esto? (Víctor, comunicación personal, 2014)

La idea es entregarles las herramientas, de que ellos entiendan qué son y después hacerles una pregunta de actualidad... (Germán, comunicación personal, 2014)

Finalmente, la triangulación de los datos obtenidos desde las personas ayudantes -en ambas carrerasconciben la ayudantía como un espacio de aprendizaje bidireccional, es decir, un espacio que potencia el aprendizaje entre ellos y sus pares (tutorados). Es en este espacio donde relevan la importancia de tener claridad sobre los contenidos a compartir con sus compañeros pares, y es en este ejercicio en donde construyen su identidad:

... si hay algo de lo que me preocupo mucho es plantearme la ayudantía como un espacio de formación para mi y los ayudantes "[estudiantes] como poder trabajar en común un tema, como un grupo de estudio, un grupo que mutuamente comparte conocimientos... (Romina, comunicación personal, 2014) 
... Mi método para mi es entender lo que estoy haciendo, si no lo entiendo, difícilmente lo puedo enseñar. No sirvo para hacer la mecánica simplemente, tengo que entender por qué y soy como obstinado con eso... (Jorge, comunicación personal, 2014)

\section{Condiciones que impactan los procesos de aprendizaje y enseñanza}

Los resultados, desde la percepción de las personas ayudantes de Ingeniería y Psicología, muestran que coordinarse con el profesorado o el equipo docente de la actividad curricular, revisar el programa y reflexionar sobre los procesos de aprender y enseñar, resultan claves para impactar de manera positiva el espacio de aprendizaje y enseñanza que se da, al interior de las ayudantías. Tal como se reporta en la Tabla 2, cada ayudante señala que estas condiciones favorecen la construcción de su aprendizaje y el de sus compañeros tutorados. Particularmente, desde la disciplina, tanto como la reflexión sobre los propios procesos de aprendizaje. 
E. Piedad Cabrera-Murcia. ¿Cómo se conceptualizan las ayudantías? La voz de las personas tutoras u...

TABLA 2.

Condiciones para que se dé una buena ayudantía.

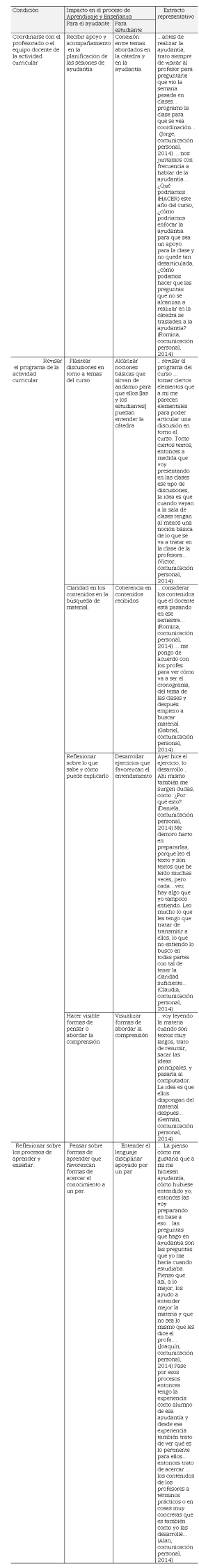




\section{Estrategias usadas en el desarrollo de la ayudantía}

La Tabla 3, muestra las estrategias usadas por ayudantes de ambas carreras para el desarrollo de las ayudantías, así como su percepción en relación con el impacto de estas en su aprendizaje como en el estudiantado tutorado. En estas, se visibiliza la potenciación de habilidades de orden superior, por ejemplo, el poder analizar, sintetizar o evaluar.

Los resultados, -obtenidos desde la triangulación de los datos- señalan el impacto que tiene el uso de estas estrategias en el desarrollo de las habilidades cognitivas y metacognitivas de ayudantes, las que enriquecen su forma de enseñar. A través del acompañamiento que hacen a sus pares, aprenden a plantear preguntas considerando el para qué de su uso. Por ejemplo, para clarificar, comprender, aclarar dudas, identificar errores de comprensión, fomentar la participación, así como para que el estudiantado piense en cómo están trabajando.

Asimismo, las personas ayudantes aprenden a identificar y plantear ejemplos que, facilitan la elaboración de conocimiento, reflexionar sobre su pensamiento y el de otros, poniéndolo en discusión y construyendo planificaciones que visibilizan los conocimientos previos. Destaca en estos hallazgos la humildad intelectual en ayudantes -habilidad relevante cuando se habla de potenciar el pensamiento crítico- ya que, no se reconocen como poseedores de la verdad, sino que se muestran a sus pares como una persona que puede equivocarse. 
E. Piedad Cabrera-Murcia. ¿Cómo se conceptualizan las ayudantías? La voz de las Personas tutoras u...

TABLA 3

Estrategias consideradas efectivas en ambas carreras

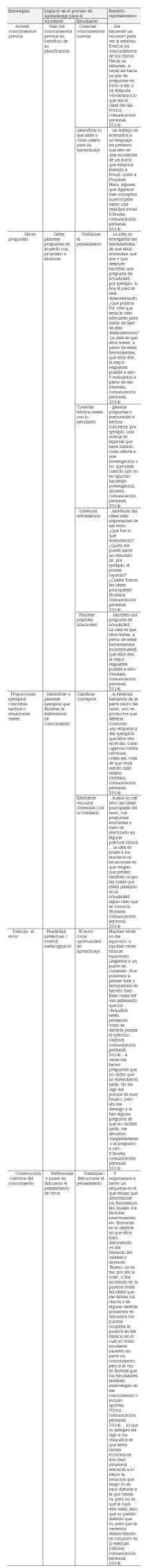


Fuente: Elaboración propia.

Los hallazgos también permiten visualizar estrategias que, no siendo comunes para las personas ayudantes de Ingeniería y Psicología, sí son de uso frecuente considerando el campo disciplinar. En ingeniería, el uso de ejercicios es clave en el diseño de las ayudantías. Con ella se promueve el pensamiento, la comprensión de procesos, tanto como de conceptos y habilidades centrales propias de la carrera. En ellos, su uso potencia habilidades como explicar procesos, modelar formas de pensar, así como considerar puntos de vista (Tabla 4).

TABLA 4

Uso de estrategia en carrera de Ingeniería

\begin{tabular}{|c|c|c|c|}
\hline \multirow[t]{2}{*}{ Estrategias } & \multicolumn{2}{|c|}{$\begin{array}{l}\text { Impacto en el proceso de } \\
\text { Aprendizaje para el: }\end{array}$} & \multirow[t]{2}{*}{ Extracto representativo } \\
\hline & Ayudante & Estudiante & \\
\hline \multirow[t]{3}{*}{$\begin{array}{l}\text { Uso } \\
\text { de } \\
\text { ejercicios. }\end{array}$} & $\begin{array}{l}\text { Considerar } \\
\text { puntos de } \\
\text { vista }\end{array}$ & $\begin{array}{l}\text { Promover el } \\
\text { pensamiento } \\
\text { (propósito) }\end{array}$ & $\begin{array}{l}\text {...todos esos ejercicios que } \\
\text { pongo son para que no } \\
\text { sean mecánicos, si no para } \\
\text { que piensen, se cuestionen } \\
\text { o debatan conmigo ... } \\
\text { (Gabriel, comunicación } \\
\text { personal, 2014) }\end{array}$ \\
\hline & \begin{tabular}{|l} 
Explicar \\
procesos
\end{tabular} & $\begin{array}{l}\text { Comprensión } \\
\text { de procesos (el } \\
\text { cómo) }\end{array}$ & $\begin{array}{l}\text { Lo que más me gusta que } \\
\text { participen, trato de hacer } \\
\text { hartas preguntas. No me } \\
\text { gusta hacer todo el rato el } \\
\text { ejercicio, trato siempre yo } \\
\text { decir: acuérdense qué se } \\
\text { hacia acá, por qué esto va } \\
\text { así. (Daniela, } \\
\text { comunicación personal, } \\
\text { 2014) }\end{array}$ \\
\hline & $\begin{array}{l}\text { Modelar } \\
\text { formas de } \\
\text { pensar }\end{array}$ & $\begin{array}{l}\text { Comprender } \\
\text { conceptos y } \\
\text { habilidades } \\
\text { centrales } \\
\text { propias de la } \\
\text { carrera }\end{array}$ & $\begin{array}{l}\text { Por ejemplo, un problema } \\
\text { de una empresa. No hay } \\
\text { una solución como única } \\
\text { para el ejercicio, sino que } \\
\text { varias formas de hacerlo y } \\
\text { depende netamente del } \\
\text { pensamiento de la } \\
\text { persona. Enseñar eso... } \\
\text { Hay un trasfondo, hay } \\
\text { una idea que es como la } \\
\text { base, pero sacar la } \\
\text { respuesta no va a ser lo } \\
\text { mismo que en un ejercicio } \\
\text { de planteamiento que del } \\
\text { otro, son todos distintos... } \\
\text { (Nicolás, comunicación } \\
\text { personal, 2014) }\end{array}$ \\
\hline
\end{tabular}

Fuente: Elaboración propia

En Psicología el construir en conjunto representaciones gráficas, reflexiones sobre los textos leídos y el presentar ejemplos que generen controversia, caracterizan el diseño de ayudantías en esta disciplina. Para las personas ayudantes, estas estrategias favorecen el aprendizaje recíproco, de tal manera que sean un apoyo para sus pares en la construcción de conocimientos y habilidades propias de la disciplina. En la Tabla 5 se muestran dichas estrategias, su impacto en el aprendizaje, desde la voz de los participantes: 
E. Piedad Cabrera-Murcia. ¿Cómo se conceptualizan las ayudantías? La voz de las personas tutoras u...

TABLA 5

Uso de estrategias en carrera de Psicología

\begin{tabular}{|c|c|c|c|}
\hline \multirow[t]{2}{*}{ Estrategias } & \multicolumn{2}{|c|}{$\begin{array}{l}\text { Impacto en el proceso de } \\
\text { Aprendizaje para el: }\end{array}$} & \multirow[t]{2}{*}{\begin{tabular}{|l|} 
Extracto \\
representativo
\end{tabular}} \\
\hline & Ayudante & & \\
\hline $\begin{array}{l}\text { Construir en } \\
\text { conjunto } \\
\text { representaciones } \\
\text { gráficas. }\end{array}$ & $\begin{array}{l}\text { Abordar } \\
\text { formas de } \\
\text { explicar } \\
\text { considerando } \\
\text { necesidades } \\
\text { de las y los } \\
\text { estudiantes. }\end{array}$ & $\begin{array}{l}\text { Estructuración } \\
\text { del } \\
\text { pensamiento y } \\
\text { de los } \\
\text { conceptos. }\end{array}$ & \begin{tabular}{|l}
.. ordeno la \\
materia en \\
esquemas. \\
Siempre me \\
sale un mapa \\
distinto... \\
Entre todos \\
vamos \\
armando un \\
mapa \\
conceptual \\
que después \\
les permite \\
estudiar... \\
(Romina, \\
comunicación \\
personal, \\
2014)
\end{tabular} \\
\hline $\begin{array}{l}\text { Reflexiones } \\
\text { sobre los textos } \\
\text { leídos. }\end{array}$ & \begin{tabular}{|l}
\multicolumn{1}{|c}{ Hacer } \\
preguntas \\
que inviten a \\
la reflexión \\
/ Acompañar \\
el proceso de \\
comprensión \\
de un texto
\end{tabular} & $\begin{array}{l}\quad \text { Entender } \\
\text { lo que el autor } \\
\text { quiere decir y } \\
\text { tomar } \\
\text { postura }\end{array}$ & 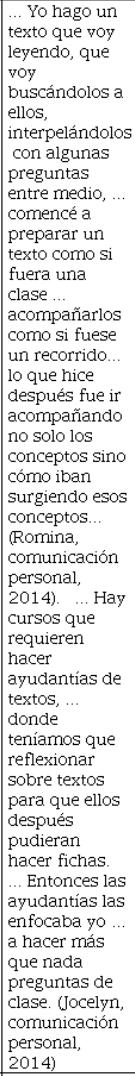 \\
\hline $\begin{array}{l}\text { Presentar } \\
\text { ejemplos que } \\
\text { generen } \\
\text { controversia }\end{array}$ & \begin{tabular}{|l|} 
Plantear \\
temas de \\
discusión / \\
mediar clases \\
dialógicas
\end{tabular} & $\begin{array}{l}\quad \text { Participar } \\
\text { evidenciando } \\
\text { su postura }\end{array}$ & $\begin{array}{l}\text { Ocupar harto } \\
\text { los ejemplos, } \\
\text { ejemplos que } \\
\text { estén de } \\
\text { moda, temas } \\
\text { que los } \\
\text { chiquillos se } \\
\text { interesen: } \\
\text { alguna } \\
\text { película o } \\
\text { cuando estaba } \\
\text { todo el } \\
\text { movimiento } \\
\text { estudiantil... } \\
\text { ¿Qué te gusta? } \\
\text { zqué opinas } \\
\text { de esto Como } \\
\text { que se pongan } \\
\text { en juego ellos, } \\
\text { como que se } \\
\text { sientan } \\
\text { involucrados, } \\
\text { porque } \\
\text { también es } \\
\text { entretenido } \\
\text { cuando uno } \\
\text { no está de } \\
\text { acuerdo, se } \\
\text { enoja y se } \\
\text { mete en la } \\
\text { conversación } \\
\text { (Romina, } \\
\text { comunicación } \\
\text { personal, } \\
\text { 2014) }\end{array}$ \\
\hline
\end{tabular}


Fuente: Elaboración propia.

\section{Análisis relacional}

La Figura 1 muestra la ayudantía como un espacio de aprendizaje recíproco demanda un conjunto de condiciones que permiten pensarla como un espacio de aprendizaje colectivo:

...Cuando preparo la ayudantía, porque por lo general me apoyo en un texto, lo leo, me planteo preguntas, cosas que quizás no entiendo también porque no tengo porque entenderlo todo... Trato de llevar preguntas en relación a lo que a mi me pasa también con eso. Trato de ayudar a los alumnos a que puedan comprender lo que leen. (Romina, comunicación personal, 2014).

A partir de este análisis relacional se evidencian ciertos elementos que pueden inhibir el que se produzca la ayudantía como un espacio de aprendizaje recíproco:

... Primero planifico la ayudantía, siempre trato de hacerlo dias antes, para poder llevar una guia, que tenga impreso cada uno. Al principio cuando empecé no lo hacía así y mucha gente se pierde... (Daniela, comunicación personal, 2014).

Es en la búsqueda de equilibrio, donde emergen estrategias de acción tendientes a minimizar el impacto que puede producir los elementos inhibidores. Por ello, cada ayudante ponen en juego estrategias de acción que impactan en el proceso de aprendizaje propio, tanto como en sus pares. Si bien puede identificarse en este análisis relacional estrategias comunes a ayudantes, sin diferenciación del campo disciplinar en el que ejercen su rol, emergen del discurso algunas estrategias propias que caracterizan el espacio de aprendizaje que se produce en su interior.

En Ingeniería, las personas ayudantes reportaron que el uso de ejercicios es clave en el diseño de sus ayudantías. Esta estrategia los acerca -ayudantes y estudiantado tutorado- a considerar puntos de vista, explicar procesos y modelar formas de pensar, haciendo uso de un lenguaje propia de la disciplina. En Psicología, aquellas consideradas claves en el ejercicio de su disciplina, son: hacer preguntas que inviten a la reflexión, acompañar el proceso de comprensión de un texto, mediar clases dialógicas a partir de la generación de controversias:

... ¿Qué piensan de esto?, quizás me parece que ahora que lo estoy viendo podría ser porque es un ejercicio importante, estar mirando lo que estás haciendo porque a veces cuando estás al frente escribes, estás desarrollando la idea, pero a veces es mucho mejor ponerte atrás y darte cuenta de las cosas que están ahipendientes. Entonces les hago una pregunta respecto al texto... (Víctor, comunicación personal, 2014).

Frente a esta forma de concebir el diseño e implementación de las ayudantías, emergen características que nutren la concepción de este espacio como uno en el que se aprende de forma recíproca. La reflexión, la construcción de conocimiento, la comprensión, la aplicación de herramientas conceptuales son elementos que se visibilizan en el relato de ayudantes de Ingeniería como de Psicología. En sus palabras:

...generar una capacidad de reflexión y capacidad de comprender lo que uno está absorbiendo... (Romina, comunicación personal, 2014) Cuando hablo de procesos creo que es la lógica que hay detrás de hacer un ejercicio. Por ejemplo, todos los cursos comienzan con la base, los primeros contenidos, los fundamentos que dicen: Este curso va a ser de esta forma. Entonces es necesario, al menos en la primera ayudantía que ellos entiendan cual es la primera lógica o por qué se están haciendo esas cosas... la idea es que sepan por qué lo están haciendo, pero esa es la lógica del procedimiento que hay detrás... (Stephanie, comunicación personal, 2014). 


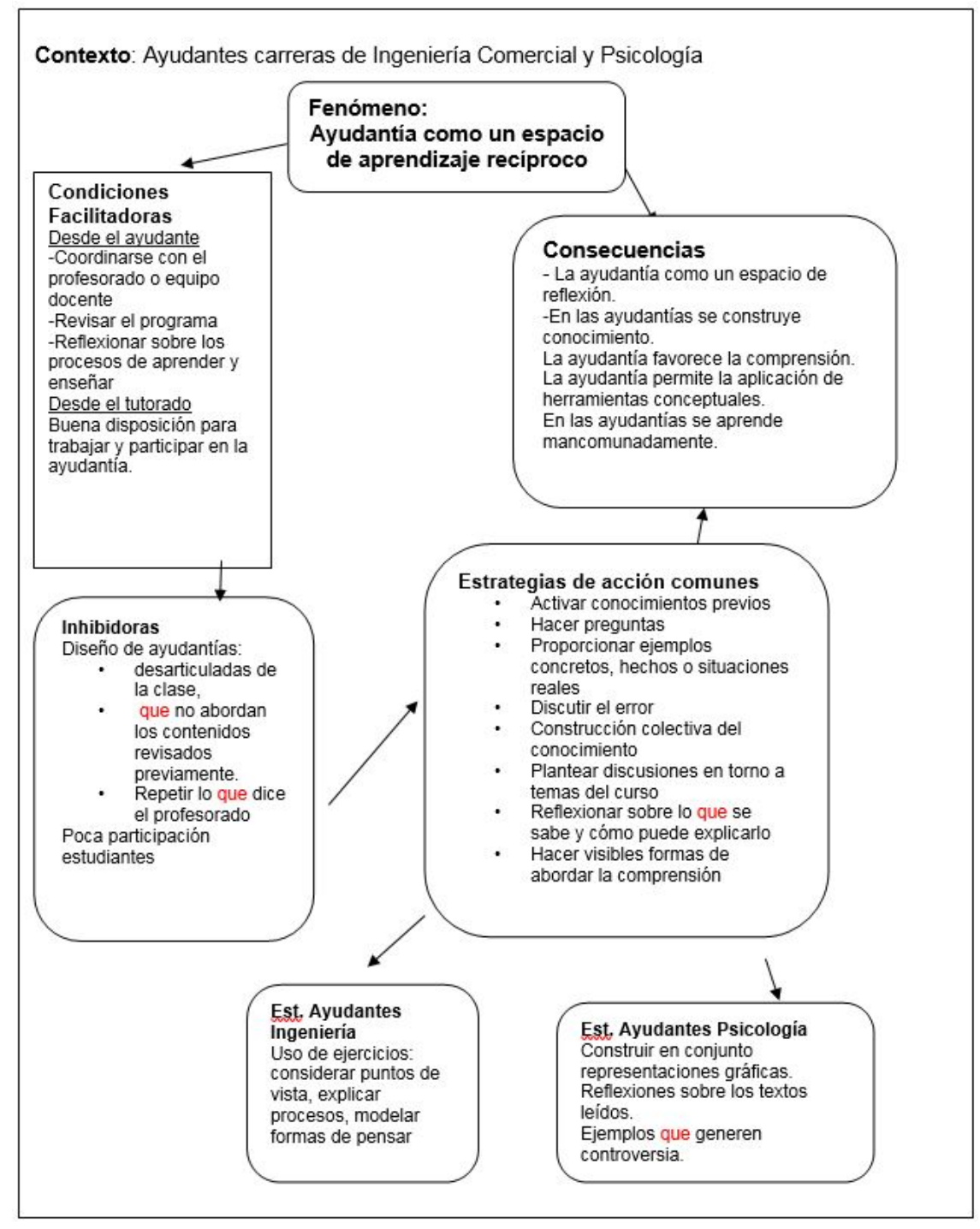

FIGURA 1

Análisis relacional del fenómeno de la ayudantía como espacio de aprendizaje recíproco Fuente: Elaboración propia

\section{DiSCUSIÓN Y CONCLUSIONES}

El estudio que se desarrolló, indicó que las ayudantías se conciben como un método de enseñanza y aprendizaje recíproco entre ayudantes y estudiantado tutorado, de reflexión, comprensión, construcción de conocimiento, aplicación de herramientas conceptuales y de experiencias de aprendizaje enriquecedoras. Es decir, espacios en los que se aprende y se enseña a aprender. Desde las voces de las personas ayudantes, este estudio permitió caracterizar las relaciones que ellos, perciben como claves en los procesos de aprender y enseñar al interior de las ayudantías.

La triangulación de la información reveló, como condiciones claves en el ejercicio de enseñar a aprender a un par: generar discusiones, plantear ejercicios que favorezcan el entendimiento y comprensión, elaborar preguntas o usar casos reales que permitan acercar el contenido al alumnado tutorado. En este contexto la noción de construcción colectiva del aprendizaje, el diálogo y la reflexión, la interacción, tanto como el rol activo de quien aprende, cobran relevancia como herramientas necesarias para aprender a aprender, así como 
aprender a enseñar. Subyace, por tanto, en la conceptualización del espacio de ayudantía, un paradigma de enseñanza-aprendizaje alejado de perspectivas más tradicionales de estos procesos.

Este marcado acento colectivo, lleva a un nivel de análisis más profundo de las estrategias, las que reflejan una forma de enseñar distintiva, que invita al estudiantado tutorado a la movilización de recursos cognitivos, en beneficio de la construcción de su propio aprendizaje. En estas, se evidencia la intencionalidad de ayudantes en acompañar al alumnado tutorado en visibilizar su pensamiento, acercar la teoría a la práctica, estableciendo vínculos entre el contenido y lo cotidiano, clarificar conceptos, el desarrollo de la comprensión y el tomar una postura frente a una situación particular. Ciertamente dicha intencionalidad, se relaciona con una forma de aprender y de enseñar que va de la mano de la comprensión, y que se hipotetiza impactaría en la forma de aprender del estudiantado que ha recibido ayudantía. Transferirla requeriría de la construcción de zonas colectivas de desarrollo próximo, tal como las nomina Engeström, que faciliten el poner en juego formas de colaboración, comprensión, construcción o complementariedad de rutas de razonamiento.

Este hecho, pone en evidencia la necesidad de experiencias formativas personalizadas, que acompañen la mejora del rol de ayudante, en beneficio de las trayectorias formativas de sus pares. Es evidente que, a la base de estos programas, se debe considerar el acompañar al ayudante en aprender desde un enfoque profundo, y para ello, debe formarse en la comprensión más que la memorización. Solo de esta manera este, será capaz de transferir a sus pares, esta forma de aproximarse al aprendizaje.

Finalmente, la muestra que se ha estudiado aquí es pequeña y se circunscribe solo a dos carreras de una universidad, aspecto que puede ser una limitante para caracterizar a cabalidad el rol del ayudante. Particularmente, porque restringe la riqueza que puede darse si se extiende a otros escenarios y desde otras disciplinas. Pese a que, el estudiantado entrevistado fue seleccionado considerando el criterio de ser evaluado como un buen ayudante se hace necesario en futuros estudios considerar la voz del alumnado que ha recibido ayudantía con las personas entrevistadas, de tal manera de poder establecer comparaciones que validen los hallazgos en relación con las condiciones y estrategias que favorecen el aprender.

Por otro lado, si bien se hizo uso de la triangulación de datos e investigadores, como estrategia, lo cual aportó regularidades en la caracterización de este espacio de enseñanza aprendizaje, a futuro es necesario el uso de diversas técnicas de recolección de los datos, que permitan minimizar debilidades o posibles sesgos al usar una sola técnica de recolección de información.

A partir de lo anterior, surgen líneas futuras de investigación desde las cuales se indague -de forma directa- sobre la práctica del ayudante, así como el impacto que puede tener, el participar en estos espacios, en los aprendizajes del estudiantado tutorado, evidenciados en el rendimiento académico. En esta misma línea, las estrategias obtenidas, pueden ser un insumo en la construcción de instrumentos que permitan caracterizar buenas prácticas en las ayudantías, o bien, un primer paso para construir programas de formación de ayudantes que puedan ser implementados y posteriormente, medir cuan efectivos han sido.

\section{Agradecimientos}

A las personas ayudantes que participaron de esta investigación, así como a las carreras de Psicología e Ingeniería Comercial de esta casa de estudios.

\section{REFERENCIAS}

Alrajhi, M. y Aldhafri, S. (2015). Peer Tutoring Effects on Omani Students' English Self-Concept. International Education Studies, 8(6), 184-193. doi https://dx.doi.org/10.5539/ies.v8n6p184

Biggs, J. (2005). Calidad del aprendizaje universitario. España: Narcea Ediciones.

Cabrera, P. (2017a). Manual para ayudantias. Construyendo aprendizaje activo entre pares en educación superior. Santiago de Chile: Universidad Alberto Hurtado. Recuperado de https://bit.ly/2LjqLxd 
Cabrera, P. (2017b). ¿Cómo diseñar ayudantías que favorezcan el aprendizaje active de los estudiantes tutoreados? La percepción del ayudante universitario. Estudios Pedagógicos, 43 (3), 47-62.

Creswell, J. (2014). Research Design. Qualitative, Quantitative and Mixed Methods Approaches. Thousand Oaks, CA: Sage

Denzin, N. (1989). Strategies of multiple triangulation. The research act: A theoretical introduction to sociological methods. New York: McGraw Hill.

Deshler, J.M. (2015). Reflections on Our First Calculus Undergraduate Teaching Assistant. Adults Learning Mathematics. An International Journal, 11(1), 59-63.

Doyle, T. (2008). Helping students learn in a learner-centered environment. A Guide to Facilitating Learning in Higher Education. United States of America: Stylus Publishing.

Engeström, Y. (2001). El aprendizaje expansivo en el trabajo: hacia una reconceptualización teórica de la actividad. Journal of Education and Work, 14(1), 1-16.

Filz, T. y Gurung, R. (2013). Student Perceptions of Undergraduate Teaching Assistants. Teaching of Psychology, 40(1), 48-51.

Flick, U. (2004). Introducción a la Investigación Cualitativa. Madrid: Ediciones Morata

Gallardo, G. y Reyes, P. (2010). Relación profesor-alumno en la universidad. Arista fundamental para el aprendizaje. Calidad de la educación, 32, 77-108

Gerton, J. (2016). Mobilizing the Forgotten Army: Improving Undergraduate Math and Science Education through Professional Development of Graduate Teaching Assistants. Bulletin of the American Physical Society. Recuperado de http://meetings.aps.org/link/BAPS.2016.MAR.V14.2

Gordon, J., Henry, P. y Michaux, D. (2013). Undergraduate Teaching Assistants: A Learner-Centered Model for Student Engagement in the First-Year Experience. International Journal of Teaching and Learning in Higher Education, 25(1), 103-109.

Hogan, T., Norcross, J., Cannon, T. y Karpiak, C. (2007). Working with and Training Undergraduates as Teaching Assistants. Teaching of Psychology, 34(3), 187-190.

Kajfez, R.L., Matusovich, H.M. y Lee, W.C. (2016). Designing Developmental Experiences for Graduate Teaching Assistants Using a Holistic Model for Motivation and Identity. The International Journal of Engineering Education, 32(3), 1208-1221.

Lindsay, K., Carlsen, B., Boaz, C. y Marshall, D. (2017). Predictors of student success in supplemental instruction courses at a medium sized woman's university. International Journal of Research in Education and Science (IJRES), 3(1), 208-217.

Malm, J., Bryngfors, L. y Mörner, L.L. (2015). The potential of Supplemental Instruction in engineering education helping new students to adjust to and succeed in University studies. European Journal of Engineering Education, 40(4), 347-365.

McKeegan, P. (1998). Using Undergraduate Teaching Assistants in a Research Methodology Course. Teaching of Psychology, 25(1), 11-14.

Melton, B. (2016). Strategies for College and University Instructional Physical Activity Program (IPAP) Directors. International Journal of Higher Education, 5(1), 292-299.

Odon, S., Ho., S. y Moore, L. (2015). An examination of the Outcomes of the Undergraduate Leadership Teaching Assistant (ULTA) Experience as a High-Impact Practice in Leadership Education. Journal of Leadership Education, 14(12), 100-117.

Organización para la Cooperación y el Desarrollo Económico (OCDE, 2009). Revisión de políticas nacionales de educación. La educación superior en Chile. Santiago, Chile: Ministerio de Educación de Chile. Recuperado de h ttp://www7.uc.cl/webpuc/piloto/pdf/informe_OECD.pdf

Philipp, S., Tretter, T.y Rich, C. (2016a). Development of Undergraduate Teaching Assistants as Effective Instructors in STEM Courses. Journal of College Science Teaching, 45(3), 74-82. 
Philipp, S., Tretter, T. y Rich, C. (2016b). Undergraduate Teaching assistant impact on student academic achievement. Electronic Journal of Science Education, 20(2), 1-13.

Sana, F., Pachai, M. y Kim, J. (2011). Training Undergraduate Teaching Assistants in a Peer Mentor Course. Transformative Dialogues. Teaching \& Learning Journal, 4(3) 1-10.

Sánchez, V., SanMartín, A. N., Mardones, C. y Fauré, J. (2017). La figura del ayudante universitario: Concepto, rol y características. Revista de Sociología, 32(1), 71-86. Recuperado de https://bit.ly/2k4lvUo

Sari, I., Çeliköz, N. y Ünal, S. (2017). The Effect of Peers Support on University Level Student's English Language Achievements. Journal of Education and Practice, 8(1), 76-81. Recuperado de https://bit.ly/2lEbmye

Strauss, A. y Corbin, J. (2002). Bases de la investigación cualitativa. Técnicas y procedimientos para desarrollar la teoría fundamentada. Colombia: Universidad de Antioquia.

Universidad Católica del Maule (2007). Reglamento del alumno ayudante. Decreto de Rectoría 19. Recuperado de ht tps://bit.ly/2k5Lr1V

Universidad de Santiago de Chile (USACH). (2009). Reglamento de Alumnos Ayudantes. Facultad de Ingeniería. Recuperado de https://bit.ly/2lZsCoX

Universidad del Desarrollo (UDD). (2014). Reglamento de Ayudantes. Recuperado de https://bit.ly/2m7GcQ7

Universidad Diego Portales (UDP). (2011). Normativa Interna Ayudantías OOCC. Recuperado de https://bit.ly/2 $\mathrm{k} 9 \mathrm{Ox} 5 \mathrm{a}$

Weidert, J., Wendorf, A., Gurung, R. y Filz, T. (2012). A survey of Graduate and Undergraduate Teaching Assistants. College Teaching, 60,95-103.

\section{BY-NC-ND}

